\title{
Edificación para un desarrollo sostenible: una actividad modal
}

\author{
Building process for sustainable development: a modal \\ activity \\ $\underline{\text { R. Tendero }}^{(*)}$, M. García de Viedma ${ }^{(* *}$
}

\section{RESUMEN}

Se proponen algunas consideraciones previas a actuaciones de cualquier tipo cuyo objetivo sea colaborar a un desarrollo humano sostenible. La justificación de estas consideraciones se deduce de declaraciones, normas y leyes de amplio consenso. Su exposición se ordena en principios que deben considerarse en cualquier actuación, aunque se han pensado para su aplicación a las fases del proceso edilicio.

Establecer con claridad estos principios permitirá comprobar la validez para el objetivo propuesto de indicadores, herramientas de diseño, programación y evaluación, procesos de ejecución, edificios, sistemas incluidos en ellos, uso durante y después de su vida útil, mantenimiento,....

Este enfoque permite la elaboración de sistemas de comprobación relacional e interactiva en el entorno físico, social, económico y de la naturaleza de las instituciones, con la finalidad de un desarrollo humano sostenible.

Esto permitirá una reflexión sobre las decisiones inherentes al proyecto de edificación con suficiente perspectiva para conseguir su colaboración con un desarrollo sostenible.

\section{SUMMARY}

The aim of this text is to suggest some considerations prior to any kind of actions which aim to contribute to human sustainable development. The rationale for these considerations can be deduced from statements, rules and laws of broad consensus. Its presentation is organized on principles that should be considered in any action, but are designed to be applied on the different parts of the building process.

It is important to establish clear principles that will enable to verify the validity of the objectives proposed on many factors as the indicators, the design tools, the planning and evaluation, the implementation processes, the buildings, the systems within them, the use during and after their useful life, the maintenance,...

This approach allows the development of both relational and interactive verification systems in the physical, social and economic environment and also on the nature of the institutions, with the purpose of a sustainable human development.

The main purpose of these facts is to make possible an approach to the decision-making related to the building project with enough perspective to get their cooperation for a sustainable development.

Keywords: Building, Sustainable Development, Human Development, Knowledge Theory, Construction.
Palabras clave: Edificación, Desarrollo Sostenible, Desarrollo Humano, Epistemología, Construcción. 
1 Se adopta la definición del Informe "Our Common Future" (1987), haciendo notar que su objetivo es el desarrollo humano y que para su consecución reconoce la necesidad de mantener un entorno saludable, entendiéndose que únicamente la conservación de un ambiente natural apto para la vida hace viable la especie humana. From A/42/427. Our Common Future: Report of the World Commission on Environment and Development Chairman Gro Harlem Brundtland Oslo, 20 March 1987.

${ }^{2}$ No se hace equivalente necesariamente crecimiento económico a desarrollo, ya que los sistemas limitados (endosfera) no pueden contener subsistemas (economía) en una permanente expansión, estando muy documentada la necesidad de poner límites al crecimiento para hacer posible que se mantenga el entorno actual. (Goodland, R and Daly, H, 1996).

${ }^{3}$ Se considera una reflexión esclarecedora la aportada sobre este tema en (Fromm, E., 1941) al diferenciar entre las necesidades fisiológicas y la necesidad de pertenencia y las tendencias y rasgos del carácter (deseos).

${ }^{4}$ Principio 4, Declaración de Río sobre el Medio Ambiente y el Desarrollo, Conferencia de las Naciones Unidas sobre el Medio Ambiente y el DesarroIlo, Río de Janeiro del 3 al 14 de junio de 1992.

${ }^{5}$ Aunque las cifras difieren según la fuente consultada se hace notar que el consumo de energía en el sector de la vivienda y los servicios puede estimarse, en la Comunidad Europea, en el $40 \%$ del consumo final (DIRECTIVA 2002/91/CE DEL PARLAMENTO EUROPEO Y DEL CONSEJO de 16 de diciembre de relativa a la eficiencia energética de los edificios, 2002), los residuos de construcción demolición podrían estar cerca de los 1.000 kg/hab y año (Plan Nacional de Residuos de Construcción y Demolición (PNRCD) 2001-2006 8, 2001), en España el consumo de materiales para construcción puede ser del $51 \%$ con respecto

\section{INTRODUCCIÓN}

El desarrollo sostenible tiene como objetivo la calidad de vida de la especie humana, y establece condiciones al uso de la Tierra, "nuestro hogar", las sociedades o la economía con este fin ${ }^{1}$ :

El desarrollo sostenible es el desarrollo que satisface las necesidades actuales sin comprometer la capacidad de futuras generaciones de satisfacer sus propias necesidades. Lo que contiene dos conceptos claves:

- el concepto de "necesidades", en particular las necesidades esenciales de los pobres del mundo, que deben tener prioridad; y

- la idea de las limitaciones impuestas por el estado de la tecnología y la organización social sobre la capacidad del ambiente de satisfacer necesidades presentes y futuras (1).

La lectura de esta definición lleva ineludiblemente a la consideración del ámbito que se otorga al concepto "necesidad humana" 2 , debe entenderse que en ella están incluidos los derechos humanos pero, en igual medida lo estarán también los derechos naturales, que permiten disfrutar de un entorno saludable sin que la actuación de otras personas limite este derecho.

En consecuencia el desarrollo humano y la necesidad de conservar su entorno es objeto de notables esfuerzos. Los instrumentos para su consecución o seguimiento resultan abundantes y son objeto de un debate muy intenso. Este desarrollo se apoya en la existencia de un acuerdo sobre sus principios generales, recogidos en documentos de amplio consenso. Se considera que la lectura atenta de estos documentos permite mantener la dirección de los esfuerzos en aras a su eficacia. En ese afán se compendian algunos de los principios en que debe basarse la acción para un desarrollo sostenible y se hace mención del documento en que se recoge. Ocasionalmente se recurre a referencias que desarrollan o anteceden a estos documentos de consenso. Para ubicar la acción en nuestro entorno se recogen algunas referencias sobre la posición europea, seleccionando los textos en función de su adaptación al fin propuesto antes que a su cronología.

Teniendo en cuenta que la actividad humana se desarrolla en un subsistema de nuestro biotopo, el desarrollo humano estará afectado en su capacidad y naturaleza por el sistema natural en que se integra ${ }^{2}$. Será preciso establecer unas condiciones a la actividad humana con el fin de mantener su desarrollo en límites sostenibles (3). Aunque el término se estable- ce y desarrolla en la búsqueda de una eficaz conservación del medioambiente, su difusión afecta desde el ámbito científico al doméstico. Esto, probablemente, se debe a que establece de un modo unívoco la relación entre desarollo humano y conservación de su entorno, afectando a la naturaleza genuinamente humana y su necesidad de pertenencia ${ }^{3}$.

A fin de alcanzar el desarrollo sostenible, la protección del medio ambiente deberá constituir parte integrante del proceso de desarrollo y no podrá considerarse en forma aislada ${ }^{4}$.

Dada su implicación en el uso de recursos y en la transformación del entorno, la colaboración de la actividad edilicia es, entre otras, fundamental para alcanzar un desarrollo sostenible ${ }^{5}$.

Dentro del proceso productivo la edificación está situada al final de una secuencia que la hace dependiente de las decisiones tomadas en otros campos de la ciencia, la industria, la economía, la ordenación del territorio, la sociología, el medioambiente,... Haciendo una simplificación, pueden enunciarse como campos tecnológico, social, económico y medioambiental

Por otra parte los edificios están en primera línea en lo que se refiere a la satisfacción de las necesidades humanas, explícitas o no, especialmente la de cobijo, entendida como soporte para el bienestar, la salud, la productividad, la espiritualidad,...

Hay que tener en cuenta que la vida útil de los edificios, aunque manifiestamente mejorable, es muy larga, mientras que la relación coste de producción-periodo de amortización puede ser menor que la de otras actividades humanas, especialmente si se comparan estos valores con los de otras actividades industriales.

Tras su gestación y construcción, durante la vida útil de un edificio, se producen dos procesos relevantes en cuanto a su implicación en un desarrollo sostenible: los derivados del uso y los derivados del mantenimiento y la adaptación a necesidades diversas. Sin duda es, en este periodo, donde se producen los mayores impactos medioambientales y la mayor repercusión social y económica, aunque no sean sólo consecuencia de la actividad edilicia, si son influidos por ella.

Las reflexiones que se proponen afectan a la edificación en su conjunto (indicadores, herramientas de diseño, programación y evaluación, procesos de ejecución, edificios, sistemas incluidos en ellos, uso durante y después de su vida útil, mantenimiento..., para conseguir optimizar su participación en un proceso que, resumiendo, parte de una 
realidad económica, medioambiental, social y tecnológica consolidada y debe satisfacer directamente las necesidades humanas en lo relativo al cobijo.

\section{PRINCIPIOS PARA LA ACCIÓN}

Los fundamentos y las reglas en las que se debe sustentar un desarrollo sostenible están en una permanente revisión en todas las áreas tecnológicas (salud, alimentación ingeniería,...) o científicas (matemáticas, físicas, químicas,...), pero esto no debe ser excusa para no actuar mientras se consolida el conocimiento; nunca ha sido así ${ }^{6}$.

La urgencia con la que se plantea la solución a los problemas ambientales y la utilización racional de los recursos hace que, en ocasiones, se postergue el análisis completo de sus implicaciones en el desarrollo dentro de un marco social y económico sostenible. Del mismo modo el debate de los principios que rigen este desarrollo sostenible y su definición desde el punto de vista fundamental y operativo deja paso a las actuaciones concretas sin tener, en ocasiones, suficientemente claras todas sus implicaciones y, lo que es más importante, su sintonía con los principios que rigen el proceso.

La búsqueda de los principios que rigen las ciencias y las actuaciones es un afán genuinamente humano y nos podemos considerar herederos de su conocimiento, independientemente de la prelación que se dé a la acción o a la reflexión.

Estos principios se justifican en consensos amplios, para poder considerarlos universales y no fruto de la endogamia o la negociación.

La Declaración de los Derechos Humanos recoge uno de estos consensos pero, por sorprendente que pueda parecer, no incluye derechos naturales que se consideraban obvios desde los primeros códices: el derecho al agua, al aire, al ambiente sano, al sol...

Los principios actuales del desarrollo sostenible tienen, probablemente, su origen en consideraciones biomiméticas o ecológicas, donde se recogen el principio precautorio, de interdependencia e interconexión, de eficiencia y mesura, de integridad, de equilibrio (dinámico),... (4). Para el fin que se pretende el método de análisis elegido no exige considerar esta génesis, está implícita en los documentos consultados ${ }^{7}$.

Teniendo en cuenta nuestro entorno social y político, se distingue entre los objetivos clave (protección ambiental, cohesión e igualdad social, prosperidad económica y cumplir con

las responsabilidades internacionales) y los principios que habrán de regir las políticas comunitarias, estableciendo entre sus principios rectores: la promoción y protección de los derechos fundamentales (incluyendo la lucha contra la discriminación y la erradicación de la pobreza global), la equidad intergeneracional, las sociedades abiertas y democráticas, la participación ciudadana, de las empresas y los interlocutores sociales, la coherencia en las políticas y la gobernanza, la integración de las políticas, el utilizar los mejores conocimientos disponibles, el principio de precaución y hacer que el contaminador pague (5).

En un ejercicio de síntesis se considera que existe consenso suficiente sobre los principios que se aportan, por lo que deben estar presentes en las prescripciones que conducen a actuaciones en edificación, materializarse en indicadores, reglas, normas, reglamentos, leyes, ... y estar sometidos a una permanente revisión, para confirmar la validez de las propuestas tecnológicas con respecto a la finalidad pretendida: conseguir un desarrollo sostenible.

Acompañando a la definición ${ }^{6}$ y el alcance del principio propuesto se recogen las referencias en declaraciones internacionales de amplio consenso ${ }^{8}(7)$, para facilitar la comprensión del sentido en que se propone cada uno de ellos (ver Tabla 1).

Tabla 1

Principios Básicos para un desarrollo sostenible y conceptos relacionados

\begin{tabular}{|l|c|c|}
\hline \multicolumn{2}{|c|}{ PRINCIPIOS PARA UN DESARROLLO SOSTENIBLE } \\
\hline \multicolumn{2}{|c|}{ PRINCIPIOS BÁSICOS } & CONCEPTOS RELACIONADOS \\
\hline 1 & UNIVERSALIDAD & $\begin{array}{l}\text { Principio de CORRESPONSABILIDAD } \\
\text { Principio de CONVERGENCIA }\end{array}$ \\
\hline 2 & EQUIDAD & $\begin{array}{l}\text { Principio de TRANSPARENCIA } \\
\text { Derecho a la INFORMACIÓN }\end{array}$ \\
\hline 3 & PARTICIPACIÓN & $\begin{array}{l}\text { Principio de LOCALIZACIÓN } \\
\text { Principio de DIVERSIDAD }\end{array}$ \\
\hline 4 & AUTONOMÍA & $\begin{array}{l}\text { Acción y Medidas Preventivas } \\
\text { Principio de PRECAUCIÓN }\end{array}$ \\
\hline 5 & CAUSALIDAD &
\end{tabular}

\subsection{Principio 1: universalidad ... respecto al sujeto}

Las necesidades humanas a las que se refiere la definición de desarrollo sostenible deben considerarse comunes a toda la especie. Aunque, en primera instancia, afecta a los individuos que comparten un colectivo o una demarcación geográfica, su extensión y difusión ha de permitir considerarlas en su carácter universal. Por ello esta condición afecta a los derechos y a las obligaciones de todos los individuos, con especial mención a su carácter intergeneracional: al total del consumo nacional (MATERIAL USE IN THE EUROPEAN UNION (1980-2000: INDICATORS AND ANALYSIS, EUROPEAN COMUNITIES 2002),...

${ }^{6}$ Se ha denominado a esta casuística la "búsqueda (o exigencia) de la prueba definitiva", argumento a menudo empleado para justificar la dilación de decisiones inevitables generalmente por razones económicas (amortizar equipos, patentes o sistemas de producción, modelos de extracción,...) o sociales (mantener una estructura favorable a determinados intereses, evitar el desarrollo de parte de la sociedad, mantener privilegios o fueros,...).

7 Para ilustrar el desarrollo actual de los principios que rigen un desarrollo sostenible pueden considerarse muchos antecedentes en áreas muy diversas (económicas, sociales, medioambientales,...) contempladas desde muy distintos puntos de vista (desarrollistas, ecologistas,...), un ejemplo afín a las propuestas realizadas puede observarse en Commoner, B. (1971).
${ }^{8}$ Se han seleccionado en función de la claridad con que manifiestan los principios que se desean explorar y el consenso que han suscitado. 
${ }^{9}$ Declaración Universal de los Derechos Humanos, 1948. Art. 1.

${ }^{10}$ Declaración de Estocolmo sobre el medio humano. Conferencia de Naciones Unidas sobre el Medio Humano, Estocolmo del 5 al 16 de junio de 1972.

${ }^{11}$ Principios 1 y 3 . Declaración de Río sobre el Medio Ambiente y el Desarrollo. Conferencia de las Naciones Unidas sobre el Medio Ambiente y el Desarrollo, Río de Janeiro del 3 al 14 de junio de 1992.

12 Principio 9. Declaración de Río sobre el Medio Ambiente y el Desarrollo. Conferencia de las Naciones Unidas sobre el Medio Ambiente y el DesarroIlo, Río de Janeiro del 3 al 14 de junio de 1992.

${ }^{13}$ Declaración Universal de los Derechos Humanos, 1948. Art. 1.

${ }^{14}$ Declaración de Estocolmo sobre el medio humano Conferencia de Naciones Unidas sobre el Medio Humano, Estocolmo del 5 al 16 de junio de 1972.

${ }^{15}$ Declaración de Río sobre el Medio Ambiente y el Desarrollo. Conferencia de las $\mathrm{Na}$ ciones Unidas sobre el Medio Ambiente y el Desarrollo, Río de Janeiro del 3 al 14 de junio de 1992, Principios 3 y 7.

${ }^{16}$ Art. 130 R apartado 2. Tratado de la Unión Europea (1992) Tit. XVI MEDIO AMBIENTE.
Todos los seres humanos nacen libres e iguales en dignidad y derechos y, dotados como están de razón y conciencia, deben comportarse fraternalmente los unos con los otros ${ }^{9}$.

El hombre tiene derecho fundamental a la libertad, la igualdad y el disfrute de condiciones de vida adecuadas en un medio de calidad tal que le permita llevar una vida digna y gozar de bienestar, y tiene la solemne obligación de proteger y mejorar el medio para las generaciones presentes y futuras... ${ }^{10}$

Los seres humanos constituyen el centro de las preocupaciones relacionadas con el desarrollo sostenible. Tienen derecho a una vida saludable y productiva en armonía con la naturaleza.

El derecho al desarrollo debe ejercerse en forma tal que responda equitativamente a las necesidades de desarrollo y ambientales de las generaciones presentes y futuras ${ }^{11}$.

\section{...del conocimiento}

También el conocimiento, la cultura, la tradición y la tecnología que permiten afrontar el reto de un desarrollo sostenible son patrimonio de la humanidad. Si los problemas son globales debe disponerse de todo este patrimonio para su resolución, globalizando las soluciones como ya lo están los problemas.

Los Estados deberían cooperar en el fortalecimiento de su propia capacidad de lograr el desarrollo sostenible, aumentando el saber científico mediante el intercambio de conocimientos científicos y tecnológicos, e intensificando el desarrollo, la adaptación, la difusión y la transferencia de tecnologías, entre éstas, tecnologías nuevas e innovadoras ${ }^{12}$.

\subsection{Principio 2: equidad}

La necesidad de trascender el principio de igualdad para que cada individuo colabore en virtud de sus cualidades, capacidades y medios, disfrutando de la satisfacción de sus necesidades en la forma que libremente elija, y, ampliando desde la satisfacción de las necesidades básicas a otras, en función de su mérito y de su esfuerzo, sin menoscabo del patrimonio natural común.

Este principio capacita para la satisfacción de las propias necesidades a la vez que limita la posibilidad de evitar la satisfacción de las necesidades de otros, sean coetáneos o no. Esto justifica la legislación para limitar la posición dominante en los mercados, el principio de proporcionalidad en la regulación del libre comercio, la consideración como patrimonio colectivo de los bienes es- casos e indispensables para satisfacer nuestras necesidades,...

Las actuaciones precisas para la reposición, lejos de pretender ser igualitarias, deben ser equitativas: proporcionales a las capacidades financieras, tecnológicas y de la presión que han ejercido sobre el medio para disponer de estas capacidades.

En lo que afecta a los derechos de subsistencia, que permiten la vida y la satisfacción de las necesidades básicas, la equidad es especialmente necesaria y su custodia una obligación de la humanidad.

Todos los seres humanos nacen libres e iguales en dignidad y derechos y, dotados como están de razón y conciencia, deben comportarse fraternalmente los unos con los otros ${ }^{13}$.

.... A este respecto, las políticas que promueven o perpetúan el apartheid, la segregación racial, la discriminación, la opresión colonial y otras formas de opresión y de dominación extranjera quedan condenadas y deben eliminarse ${ }^{14}$.

El derecho al desarrollo debe ejercerse en forma tal que responda equitativamente a las necesidades de desarrollo y ambientales de las generaciones presentes y futuras.

... En vista de que han contribuido en distinta medida a la degradación del medio ambiente mundial, los Estados tienen responsabilidades comunes pero diferenciadas. Los países desarrollados reconocen la responsabilidad que les cabe en la búsqueda internacional del desarrollo sostenible, en vista de las presiones que sus sociedades ejercen en el medio ambiente mundial y de las tecnologías y los recursos financieros de que disponen ${ }^{15}$.

Especialmente en el caso de la reposición de los daños causados por una actividad o mal uso, la respuesta colectiva a los problemas debe ser proporcional a la responsabilidad (principio de corresponsabilidad), estableciéndose mecanismos que permitan afectar la capacidad de disfrutar de los bienes naturales con el coste preciso para mantener en medioambiente y su potencial para satisfacer las necesidades humanas:

(...en el ámbito del medio ambiente...)...Se basará en...el principio de quien contamina paga... ${ }^{16}$.

Las autoridades nacionales deberían procurar fomentar la internalización de los costos ambientales y el uso de instrumentos económicos, teniendo en cuenta el criterio de que el que contamina debe, en principio, cargar con los costos de la contaminación, teniendo 
debidamente en cuenta el interés público y sin distorsionar el comercio ni las inversiones internacionales ${ }^{17}$.

COHESIÓN E IGUALDAD SOCIAL: fomentar una sociedad democrática, socialmente incluyente, cohesionada, sana, segura y justa que respete los derechos fundamentales y la diversidad cultural, que ofrezca las mismas oportunidades para todos sus miembros y combata la discriminación en todas sus formas.

EQUIDAD INTRA E INTERGENERACIONAL: hacer frente a las necesidades de los habitantes de la UE y del resto del planeta sin poner en peligro la capacidad de las futuras generaciones para cubrir las suyas ${ }^{18}$.

Forma parte esencial de este principio la necesidad de evitar conductas injustas a sabiendas, especialmente en lo relacionado a la corrupción, la extorsión o el soborno.

"Las empresas deberán trabajar contra la corrupción en todas sus formas, incluidas extorsión y soborno." 19

En el ámbito económico hace referencia a la moderación al establecer el precio de las cosas y en no colaborar a hacer inalcanzables los bienes necesarios, en el social establece el derecho a disfrutar de los mismos derechos y obligaciones y en relación al entorno permite considerarlo patrimonio común y, como tal, responsabilidad común.

\section{Principio de CONVERGENCIA}

Actualmente y dado el progresivo deterioro de la equidad ${ }^{20}$, sólo puede establecerse un camino hacia ella a través de la convergencia, entendida como la reversión de este proceso. Esto tiene repercusiones económicas, sociales y medioambientales. Mantener o acrecentar las diferencias no podría desde este punto de vista considerarse sostenible. A este principio le corresponde la responsabilidad común de poner límites al crecimiento económico, que no al desarrollo humano, dado que, como ya se ha comentado el subsistema económico, donde se producen las transacciones y los bienes tienen un precio que permite su intercambio, esta incluido en el sistema global: por lo que el biotopo humano no es infinito.

La inclusión de este principio se realiza siendo consciente de que, a diferencia de los otros, no está amparado en un consenso tan generalizado. Esto puede deberse a que es mucho más concreto, se mide con más facilidad y tiene consecuencias inmediatas en nuestra actividad, encontrándose en el límite entre ser considerado un principio o una consecuencia, evaluable con los indicadores o variables que miden el desarrollo.

Su inclusión se debe a la necesidad de limitar el crecimiento asimétrico actual y se contrapone con la superstición de que la calidad de vida, el crecimiento permanente de la economía, la "renta per cápita" y el desarrollo humano son sinónimos o directamente proporcionales.

Todos los Estados y todas las personas deberán cooperar en la tarea esencial de erradicar la pobreza como requisito indispensable del desarrollo sostenible, a fin de reducir las disparidades en los niveles de vida y responder mejor a las necesidades de la mayoría de los pueblos del mundo.

Se deberá dar especial prioridad a la situación y las necesidades especiales de los países en desarrollo, en particular los países menos adelantados y los más vulnerables desde el punto de vista ambiental. En las medidas internacionales que se adopten con respecto al medio ambiente y al desarrollo también se deberían tener en cuenta los intereses y las necesidades de todos los países ${ }^{21}$.

\section{Principio de CORRESPONSABILIDAD (solidaridad)}

Nuestra relación con el entorno la define el ser compartida con otros y que nuestros actos entrañan consecuencias que también son compartidas por otros. Transciende al concepto de solidaridad que supone la adhesión circunstancial a la causa o a la empresa de otros, con un componente de generosidad que no sería de aplicación cuando se ha colaborado a causar el daño y el beneficio que conlleva repercutirá en cada uno. Si se entendiese la solidaridad con la acepción empleada en ciencias jurídicas, más consecuente con su raíz etimológica, como modo de derecho u obligación "in solidum", donde se unen inseparablemente los destinos de dos o más personas, físicas o jurídicas, sería más acorde con la Responsabilidad Compartida que indica la Corresponsabilidad.

La corresponsabilidad supone, por tanto, responder de algo compartiéndolo con otros, como reconocer y aceptar las consecuencias de un hecho realizado libremente, del que las sociedades mas desarrolladas han sacado provecho y ventaja competitiva.

... Los países desarrollados reconocen la responsabilidad que les cabe en la búsqueda internacional del desarrollo sostenible, en vista de las presiones que sus sociedades ejercen en el medio ambiente mundial y de las tecnologías y los recursos financieros de que disponen ${ }^{22}$.
${ }^{17}$ Principio 16. Declaración de Río sobre el Medio Ambiente y el Desarrollo. Conferencia de las Naciones Unidas sobre el Medio Ambiente y el Desarrollo, Río de Janeiro del 3 al 14 de junio de 1992.

${ }^{18}$ Proyecto de Declaración sobre los Principios Rectores del Desarrollo Sostenible. Comunicación de la Comisión al Consejo y al Parlamento Europeo, Bruselas, 25.5.2005.

19 PACTO MUNDIAL. RESOLU CIÓN SOBRE LA INCLUSIÓN DE UN DÉCIMO PRINCIPIO CONTRA LA CORRUPCIÓN 30 DE MAYO DE 2004, propuesta del Secretario General de la ONU Kofi Annan.

${ }^{20}$ Esta afirmación puede observarse mediante indicadores, tales como la calidad de vida, la huella ecológica, la renta per cápita, ... u otros, pero, en cualquier caso, la conclusión es la misma.

${ }^{21}$ Principios 5 y 6 . Declaración de Río sobre el Medio Ambiente y el Desarrollo. Conferencia de las Naciones Unidas sobre el Medio Ambiente y el Desarrollo, Río de Janeiro del 3 al 14 de junio de 1992

22 Principio 7. Declaración de Río sobre el Medio Ambiente y el Desarrollo. Conferencia de las Naciones Unidas sobre el Medio Ambiente y el Desarrollo, Río de Janeiro del 3 al 14 de junio de 1992. 
${ }^{23}$ Proyecto de Declaración sobre los Principios Rectores del Desarrollo Sostenible. Comunicación de la Comisión al Consejo y al Parlamento Europeo, Bruselas, 25.5.2005.

${ }^{24}$ Tratado de la Unión Europea Diario Oficial $n^{\circ}$ C 191 de 29 de julio de 1992 TíTULO XVI MEDIO AMBIENTE Art. $130 \mathrm{R}$ apartado 2.

${ }^{25}$ Proyecto de Declaración sobre los Principios Rectores del Desarrollo Sostenible. Comunicación de la Comisión al Consejo y al Parlamento Europeo, Bruselas, 25.5.2005.

${ }^{26}$ La meta se define en el mismo texto como: la defensa y el mejoramiento del medio humano para las generaciones presentes y futuras se ha convertido en meta imperiosa de la humanidad, que ha de perseguirse al mismo tiempo que las metas fundamentales ya establecidas de la paz y el desarrollo económico y social en todo el mundo, y de conformidad con ellas. Declaración de Estocolmo sobre el Medio Humano. Conferencia de Naciones Unidas sobre el Medio Humano, Estocolmo del 5 al 16 de junio de 1972.

${ }^{27}$ Declaración de Estocolmo sobre el Medio Humano (1972).

${ }^{28}$ Principio 10. Declaración de Río sobre el Medio Ambiente y el Desarrollo. Conferencia de las Naciones Unidas sobre el Medio Ambiente y el DesarroIlo, Río de Janeiro del 3 al 14 de junio de 1992.

${ }^{29}$ Principios 20,21 y 22. Declaración de Río sobre el Medio Ambiente y el Desarrollo. Conferencia de las Naciones Unidas sobre el Medio Ambiente y el Desarrollo, Río de Janeiro del 3 al 14 de junio de 1992.

30 Proyecto de Declaración sobre los Principios Rectores del Desarrollo Sostenible COMUNICACIÓN DE LA COMISIÓN AL CONSEJO Y AL PARLAMENTO EUROPEO, Bruselas, 25.5.2005.
CUMPLIR CON NUESTRAS RESPONSABILIDADES INTERNACIONALES: favorecer el establecimiento y defender la estabilidad de instituciones democráticas en todo el mundo, basadas en la paz, la seguridad y la libertad. Contribuir de forma activa al desarrollo sostenible del planeta y garantizar que las políticas internas y externas de la Unión Europea son coherentes con el desarrollo sostenible del planeta y se ajustan a los compromisos internacionales ${ }^{23}$.

Esta corresponsabilidad no puede limitar la responsabilidad de los agentes directamente causantes de los impactos medioambientales, sociales y económicos independientemente del entorno en que se produzcan. Se debe obligar al causante, en la medida de sus posibilidades, a restituir el orden natural a una situación anterior al daño causado; aunque, en la práctica, suelen proponerse medidas compensatorias o valoraciones económicas del "coste natural" siendo preciso comprobar que éstas limitan o corrigen la degradación de la capacidad de satisfacer las necesidades humanas:

(...en el ámbito del medio ambiente...)...Se basará...en el principio de corrección de los atentados al medio ambiente,... y en el principio de quien contamina paga... ${ }^{24}$.

HACER QUE EL CONTAMINADOR PAGUE: garantizar que los precios reflejan los costes reales para la sociedad de las actividades de producción y consumo y que los contaminadores pagan los daños que causan a la salud pública y al medio ambiente ${ }^{25}$.

\subsection{Principio 3: participación}

Las actuaciones se realizan por y para las personas y la satisfacción de sus necesidades como especie. La participación se habrá de considerar en todas las fases del proceso: establecimiento de su necesidad, definición, ejecución, mantenimiento, la forma de uso, durante su vida útil y cuando ésta haya terminado,...

Para llegar a esa meta ${ }^{26}$ será preciso que ciudadanos y comunidades, empresas e instituciones, en todos los planos, acepten las responsabilidades que les incumben y que todos ellos participen equitativamente en la labor común. Hombres de toda condición y organizaciones de diferente índole plasmarán, con la aportación de sus propios valores y la suma de sus actividades, el medio ambiente del futuro ${ }^{27}$.

El mejor modo de tratar las cuestiones ambientales es con la participación de todos los ciudadanos interesados, en el nivel que corresponda ${ }^{28}$.

\section{... respecto al sujeto}

Este principio afecta a los individuos y colectivos independientemente de su voluntad, de su interés o de su conocimiento, ya que las decisiones les afectan y su intervención afecta a los resultados. No existirán exclusiones en razón al género, la edad, la cultura, naturaleza (jurídica,...)

Las mujeres desempeñan un papel fundamental en la ordenación del medio ambiente y en el desarrollo. Es, por tanto, imprescindible contar con su plena participación para lograr el desarrollo sostenible.

Debería movilizarse la creatividad, los ideales y el valor de los jóvenes del mundo para forjar una alianza mundial orientada a lograr el desarrollo sostenible y asegurar un mejor futuro para todos.

Las poblaciones indigenas y sus comunidades, así como otras comunidades locales, desempeñan un papel fundamental en la ordenación del medio ambiente y en el desarrollo debido a sus conocimientos y prácticas tradicionales. Los Estados deberían reconocer y apoyar debidamente su identidad, cultura e intereses y hacer posible su participación efectiva en el logro del desarrollo sostenible ${ }^{29}$.

UNA SOCIEDAD ABIERTA Y DEMOCRÁTICA: garantizar el derecho de los ciudadanos al acceso a la información y a la justicia. Desarrollar canales adecuados de consulta y de participación de todas las asociaciones y partes interesadas.

PARTICIPACIÓN CIUDADANA: impulsar la participación ciudadana en los procesos de toma de decisiones. Aumentar la educación y la sensibilización de la opinión pública en materia de desarrollo sostenible. Informar a los ciudadanos del impacto de su modo de vida en el medio ambiente y de las alternativas más sostenibles a su disposición.

PARTICIPACIÓN DE LAS EMPRESAS Y DE LOS INTERLOCUTORES SOCIALES: mejorar el diálogo social, aumentar la responsabilidad social de las empresas y fomentar las asociaciones entre el sector público y el privado para lograr una mayor cooperación y el establecimiento de responsabilidades comunes para conseguir una producción y consumo sostenibles ${ }^{30}$.

\section{... respecto a la repercusión de los actos}

Al tomar parte en las actuaciones antrópicas, en su doble vertiente de acción y de efecto, se afecta a la naturaleza y disponibilidad de los medios naturales para el desarrollo humano. 
. .la capacidad del hombre de transformar lo que le rodea, utilizada con discernimiento, puede llevar a todos los pueblos los beneficios del desarrollo y ofrecerles la oportunidad de ennoblecer su existencia. Aplicado errónea o imprudentemente, el mismo poder puede causar daños incalculables al ser humano y a su medio ${ }^{31}$.

...Deberá proporcionarse acceso efectivo a los procedimientos judiciales y administrativos, entre éstos el resarcimiento de daños y los recursos pertinentes ${ }^{32}$.

Las actuaciones entrañan consecuencias, permitiendo disfrutar de beneficios, formando una comunidad que participa de un capital en una empresa común:

El hombre es a la vez obra y artífice del medio que le rodea, el cual le da el sustento material y le brinda la oportunidad de desarrollarse intelectual, moral, social y espiritualmente ${ }^{33}$.

El hombre tiene el derecho fundamental a la libertad, la igualdad y el disfrute de condiciones de vida adecuadas en un medio de calidad tal que le permita llevar una vida digna y gozar de bienestar, y tiene la solemne obligación de proteger y mejorar el medio para las generaciones presentes y futuras ${ }^{34}$.

Del mismo modo estas actuaciones comprometen este capital y, en aras al bien común, exigen una actitud respetuosa para con su naturaleza y disponibilidad, obligando a simultanear su disfrute con su conservación:

El hombre tiene la responsabilidad especial de preservar y administrar juiciosamente el patrimonio de la flora y fauna silvestres y su hábitat, que se encuentra actualmente en grave peligro por una combinación de factores adversos. En consecuencia, al planificar el desarrollo económico debe atribuirse importancia a la conservación de la Naturaleza, incluidas la flora y fauna silvestres.

Los recursos no renovables de la Tierra deben emplearse de forma que se evite el peligro de su futuro agotamiento y se asegure que toda la humanidad comparte los beneficios de tal empleo ${ }^{35}$.

\section{Principio de TRANSPARENCIA}

Podríamos denominar transparencia, a la disponibilidad de información, en el sentido de mostrar una imagen clara, adaptada a las necesidades y capacidades del usuario, especialmente durante el tiempo abierto ${ }^{36}$.

Los Estados deberían cooperar en el fortalecimiento de su propia capacidad de lograr el desarrollo sostenible, aumentando el saber científico mediante el intercambio de conocimientos científicos y tecnológicos, e intensificando el desarrollo, la adaptación, la difusión y la transferencia de tecnologías, entre éstas, tecnologías nuevas e innovadoras ${ }^{37}$.

\section{... en la información}

Recibir parte, noticia o aviso de las actuaciones, es consustancial a la participación, permitiendo orientar los esfuerzos aprovechando la sinergia. Dentro de este ámbito, se considera que tiene carácter informativo si da forma sustancial a algo, constituyéndose en un conjunto organizado de datos y generando conocimiento. Esta información será eficaz si se adapta a la capacidad de los individuos y colectivos para recibir y entender este conocimiento y actuar en consecuencia.

...toda persona deberá tener acceso adecuado a la información sobre el medio ambiente de que dispongan las autoridades públicas, incluida la información sobre los materiales y las actividades que encierran peligro en sus comunidades..

... Los Estados deberán facilitar y fomentar la sensibilización y la participación de la población poniendo la información a disposición de todos.

Los Estados deberían reconocer y apoyar debidamente su identidad ${ }^{38}$, cultura e intereses y hacer posible su participación efectiva en el logro del desarrollo sostenible.

Los Estados deberán notificar inmediatamente a otros Estados de los desastres naturales $u$ otras situaciones de emergencia que puedan producir efectos nocivos súbitos en el medio ambiente de esos Estados.

Los Estados deberán proporcionar la información pertinente, y notificar previamente y en forma oportuna, a los Estados que posiblemente resulten afectados por actividades que puedan tener considerables efectos ambientales transfronterizos adversos, y deberán celebrar consultas con esos Estados en una fecha temprana y de buena fe $\mathrm{e}^{39}$.

En nuestro entorno próximo la información que se debe compartir aún va más lejos si se consideran los conocimientos sociales, económicos, tecnológicos y científicos; salvaguardando los derechos de autor de las personas que más colaboren a este conocimiento, en la misma media en que estos son deudores de la cultura colectiva:

Toda persona tiene derecho a tomar parte libremente en la vida cultural de la comuni-
${ }^{31}$ Declaración de Estocolmo sobre el medio humano. Conferencia de Naciones Unidas sobre el Medio Humano, Estocolmo del 5 al 16 de junio de 1972.

32 Principio 10. Declaración de Río sobre el Medio Ambiente y el Desarrollo. Conferencia de las Naciones Unidas sobre el Medio Ambiente y el DesarroIlo, Río de Janeiro del 3 al 14 de junio de 1992.

${ }^{33}$ Declaración de Estocolmo sobre el medio humano. Conferencia de Naciones Unidas sobre el Medio Humano, Estocolmo del 5 al 16 de junio de 1972.

${ }^{34}$ Principio 1. Declaración de Río sobre el Medio Ambiente y el Desarrollo. Conferencia de las Naciones Unidas sobre el Medio Ambiente y el DesarroIlo, Río de Janeiro del 3 al 14 de junio de 1992.

35 Principios 4 y 5 . Declaración de Río sobre el Medio Ambiente y el Desarrollo. Conferencia de las Naciones Unidas sobre el Medio Ambiente y el Desarrollo, Río de Janeiro del 3 al 14 de junio de 1992.

${ }^{36}$ Tiempo durante el cual la participación es posible.

${ }^{37}$ Principio 9. Declaración de Río sobre el Medio Ambiente y el Desarrollo. Conferencia de las Naciones Unidas sobre el Medio Ambiente y el Desarrollo, Río de Janeiro del 3 al 14 de junio de 1992.

${ }^{38}$ Se refiere a "Las poblaciones indígenas y sus comunidades, así como otras comunidades locales".

${ }^{39}$ Principios 10, 18, 19 y 22. Declaración de Río sobre el Medio Ambiente y el DesarroIlo, 1992. 
${ }^{40}$ Declaración Universal de los Derechos Humanos, 1948. Art. 27.1

${ }^{41}$ Proyecto de Declaración sobre los Principios Rectores del Desarrollo Sostenible COMUNICACIÓN DE LA COMISIÓN AL CONSEJO Y AL PARLAMENTO EUROPEO, Bruselas, 25.5.2005.

${ }^{42}$ Declaración Universal de los Derechos Humanos, 1948. Art. 27.2.

${ }^{43}$ La posición Europea actualizada se recoge en 9.2.2005 "COMUNICACIÓN DE LA COMISIÓN AL CONSEJO Y AL PARLAMENTO EUROPEO Revisión en 2005 de la Estrategia de la Unión Europea para un desarrollo sostenible: Primer balance y orientaciones futuras 5.5 .

${ }^{44}$ En su acepción jurídica puede considerarse como la autonomía de voluntad.

${ }^{45}$ Declaración de Río sobre el Medio Ambiente y el DesarroIlo. Conferencia de las Naciones Unidas sobre el Medio Ambiente y el Desarrollo, Río de Janeiro del 3 al 14 de junio de 1992, Principio 9.

${ }^{46}$ Principio 22. Declaración de Río sobre el Medio Ambiente y el Desarrollo. Conferencia de las Naciones Unidas sobre el Medio Ambiente y el DesarroIlo, Río de Janeiro del 3 al 14 de junio de 1992.

${ }^{47}$ Principio 22. Declaración de Río sobre el Medio Ambiente y el Desarrollo. Conferencia de las Naciones Unidas sobre el Medio Ambiente y el DesarroIlo, Río de Janeiro del 3 al 14 de junio de 1992. dad, a gozar de las artes y a participar en el progreso científico y en los beneficios que de él resulten ${ }^{40}$.

UTILIZAR LOS MEJORES CONOCIMIENTOS DISPONIBLES: garantizar el desarrollo, evaluación y ejecución de las políticas de acuerdo con los mejores conocimientos disponibles. Garantizar que dichas políticas son rentables y viables económicamente ${ }^{41}$.

Toda persona tiene derecho a la protección de los intereses morales y materiales que le correspondan por razón de las producciones científicas, literarias o artísticas de que sea autora $^{42}$.

\section{... en la difusión de resultados}

La fuente de información más valiosa deben ser las actuaciones que pretenden colaborar a un desarrollo sostenible, mediante su seguimiento, el análisis de los resultados y su difusión, tanto en lo relativo al conocimiento como a los avances sociales, económicos, tecnológicos o científicos. Ninguna actuación aislada colabora por si sola en un desarrollo sostenible, salvo cuando la especie humana pueda disponer de esta información y replicarla adaptándola a sus necesidades.

Garantizar un seguimiento eficaz ....El seguimiento se efectuará principalmente sobre la base de los indicadores de desarrollo sostenible establecidos por la Comisión, que se fundarán, entre otras cosas, en los diversos indicadores elaborados en el marco de los procesos de las políticas sectoriales y en la serie de indicadores estructurales que sintetizan estos últimos ${ }^{43}$.

\subsection{Principio 4: autonomía}

La cualidad de las asociaciones humanas que ha permitido explorar más soluciones diferentes para su adaptación al entorno ha sido, probablemente, la autonomía. Especialmente si se entiende como el derecho de las personas para definir el contenido de sus relaciones conforme a sus intereses ${ }^{44}$ (sin otros límites que los derivados de las leyes imperativas, de la moral y del orden público).

Si no es excluyente, otros individuos pueden imitar las estrategias de los más afortunados y incluso agregarse a ellos formando colectividades mayores. Esto permite que, personal y colectivamente, se tenga la capacidad de someterse a las normas sin exigencias externas o internas, permitiendo reflexionar y actuar en consecuencia a su aprendizaje y su aplicación. Especialmente si está asociado al principio de participación.
Los Estados deberían cooperar en el fortalecimiento de su propia capacidad de lograr el desarrollo sostenible, aumentando el saber científico mediante el intercambio de conocimientos científicos y tecnológicos, e intensificando el desarrollo, la adaptación, la difusión y la transferencia de tecnologías, entre éstas, tecnologías nuevas e innovadoras ${ }^{45}$.

Debe respetarse el derecho a decidir sobre la naturaleza del desarrollo de cada comunidad. Tomando relevancia, en este aspecto, la consideración de que, sociedades y personas, se encuentran en un cambio constante siendo solo posible concebir su existencia en un estado de permanente adaptación.

Esta autonomía de las personas y las sociedades aumenta su capacidad para superar las crisis con soluciones nuevas o tradicionales, generar nuevas estructuras o reutilizar en parte las antiguas, evitando su colapso.

Las poblaciones indígenas y sus comunidades, así como otras comunidades locales, desempeñan un papel fundamental en la ordenación del medio ambiente y en el desarrollo debido a sus conocimientos y prácticas tradicionales... ${ }^{46}$

\section{Principio de DIVERSIDAD}

Los individuos y las colectividades con necesidades diferentes deben poder disponer de medios diferentes para conseguir sus objetivos. Este principio en cuanto a las necesidades fisiológicas básicas (impulsos biológicos) es muy similar para todos los individuos, sin embargo es de aplicación a la necesidad básica de pertenencia o a la satisfacción de otras necesidades (tendencias o rasgos del carácter) específicas a un determinado grupo de individuos (2).

... Los Estados deberían reconocer y apoyar debidamente su identidad, cultura e intereses y hacer posible su participación efectiva en el logro del desarrollo sostenible ${ }^{47}$.

\section{Principio de LOCALIZACIÓN}

El desplazamiento de las consecuencias ambientales, económicas o sociales de una actividad debe someterse a una revisión crítica. Colaborarán con un desarrollo más sostenible aquellas actividades que localicen las consecuencias junto con los beneficios evitando que su deslocalización permita una repercusión desequilibrada de cargas y beneficios.

Esto exige considerar un reparto equitativo de los beneficios y cargas evitando que los efectos de los desequilibrios sociales o económicos causen una desigual satisfacción de 
las necesidades presentes y futuras de los grupos sociales.

Los Estados deberían cooperar efectivamente para desalentar o evitar la reubicación y la transferencia a otros Estados de cualesquiera actividades y sustancias que causen degradación ambiental grave o se consideren nocivas para la salud humana ${ }^{48}$.

Del mismo modo debe establecerse una relación equilibrada entre los costes sociales, económicos y medioambientales provocados por el transporte frente a sus posibilidades de optimizar los procesos tecnológicos, los intercambios sociales y culturales, la complementariedad económica y productiva, ...

... Las medidas de política comercial con fines ambientales no deberían constituir un medio de discriminación arbitraria o injustificable ni una restricción velada del comercio internacional. Se debería evitar tomar medidas unilaterales para solucionar los problemas ambientales que se producen fuera de la jurisdicción del país importador... ${ }^{49}$

\subsection{Principio 5: causalidad}

Relacionar las causas y los efectos es el procedimiento científico mas utilizado para progresar en el conocimiento. Aunque puede presentar diversas formas en su exposición, viene a establecer cómo todo efecto tiene una causa y que, bajo las mismas condiciones, las mismas causas preceden al mismo efecto.

En el ámbito del desarrollo sostenible se aplica a la necesidad de conocer y actuar sobre las causas para corregir sus efectos.

No será suficiente con modificar el entorno en que las causas tienen lugar ni actuar exclusivamente sobre sus consecuencias, aunque resulten necesarias medidas paliativas o correctivas aplicadas a estos efectos: solo la corrección de las causas puede ser considerada una medida eficaz.

Este principio se puede considerar implícito en todos los referidos a la necesidad de mejorar nuestro conocimiento científico.

Por otra parte este principio se aplica al ámbito social, económico y medioambiental, a pesar de la dificultad en reproducir experimentalmente estados que permitan asociar unívocamente las causas a los efectos.

(...en el ámbito del medio ambiente...)... Se basará...en el principio de corrección de los atentados al medio ambiente, preferentemente en la fuente misma, ... ${ }^{50}$

\section{Principio de PRECAUCIÓN (cautela)}

Las actuaciones en un medio de naturaleza e interrelaciones desconocidas, deben realizarse con precaución. Si se demuestra que las prácticas actuales producen o pueden producir una afección sobre el entorno que tenga como consecuencia una modificación sustancial de su naturaleza debe valorarse, de una parte, la existencia de otras prácticas que eviten estos efectos $y$, de otra, si colaboran realmente en satisfacer necesidades básicas de los individuos o las colectividades (Principio de cautela).

Deben preverse las consecuencias de las actuaciones, realizándose tantas pruebas como sean precisas para evitar riesgos tanto en los individuos, en las sociedades, como en el entorno (Acción preventiva) ${ }^{51}$ :

Con el fin de proteger el medio ambiente, los Estados deberán aplicar ampliamente el criterio de precaución conforme a sus capacidades. Cuando haya peligro de daño grave o irreversible, la falta de certeza científica absoluta no deberá utilizarse como razón para postergar la adopción de medidas eficaces en función de los costos para impedir la degradación del medio ambiente ${ }^{52}$.

(...en el ámbito del medio ambiente...) ... Se basará en los principios de cautela y de acción preventiva, ... ${ }^{53}$

...considera que se debe recurrir al principio de cautela cuando se determine la posibilidad de efectos nocivos para la salud o el medio ambiente y una evaluación científica preliminar, a tenor de los datos disponibles, no permita establecer con certeza el nivel de riesgo ${ }^{54}$.

PRINCIPIO DE PRECAUCIÓN: Observar el principio de precaución en caso de que existan dudas científicas razonables para evitar posibles daños a la salud pública o el medio ambiente... ${ }^{55}$

\section{Acción preventiva}

Debe observarse que asociado al concepto del principio de precaución, que limita las actuaciones en función del conocimiento existente sobre sus consecuencias, se establece la necesidad de realizar acciones y tomar medidas para prevenir los posibles daños. Estas dos vertientes de la misma línea de actuación indica la necesidad de que la limitación de actividad se asocie a la capacidad de actuar y la necesidad de potenciar acciones que prevengan o hagan innecesarias las actuaciones sobre las que se aplica el principio de precaución.
${ }^{48}$ Principio 14. Declaración de Río sobre el Medio Ambiente y el Desarrollo. Conferencia de las Naciones Unidas sobre el Medio Ambiente y el Desarrollo, Río de Janeiro del 3 al 14 de junio de 1992.

${ }^{49}$ Principio 12. Declaración de Río sobre el Medio Ambiente y el Desarrollo. Conferencia de las Naciones Unidas sobre el Medio Ambiente y el Desarrollo, Río de Janeiro del 3 al 14 de junio de 1992.

${ }^{50}$ Art. $130 \mathrm{R}$ apartado 2. Tratado de la Unión Europea Diario Oficial $n^{\circ}$ C 191 de 29 de julio de 1992 TíTULO XVI MEDIO AMBIENTE.

${ }^{51}$ En el ámbito europeo se hace hincapié en la necesidad y naturaleza de la evaluación científica y en la necesidad de revisión a la luz de la evolución del conocimiento, estableciendo la necesidad de transparencia, participación y haciéndolo compatible con el principio de proporcionalidad. Se establecerán sus costes medioambientales y sociales $y$, si es posible, económicos. TRATADO DE NIZA. CONSEJO EUROPEO DE NIZA 7-10 de diciembre de 2000 CONCLUSIONES DE LA PRESIDENCIA.

${ }^{52}$ Principio 15. Declaración de Río sobre el Medio Ambiente y el Desarrollo. Conferencia de las Naciones Unidas sobre el Medio Ambiente y el Desarrollo, Río de Janeiro del 3 al 14 de junio de 1992.

53 Tratado de la Unión Europea (1992) Art. $130 \mathrm{R}$ apartado 2.

${ }^{54}$ ANEXO III - Resolución del Consejo sobre el recurso al principio de cautela. Tratado de Niza. Consejo Europeo de Niza 7-10 de diciembre de 2000 Conclusiones de la Presidencia.

${ }^{55}$ Proyecto de Declaración sobre los Principios Rectores del Desarrollo Sostenible COMUNICACIÓN DE LA COMISIÓN AL CONSEJO Y AL PARLAMENTO EUROPEO, Bruselas, 25.5.2005. 
${ }^{56}$ Proyecto de Declaración sobre los Principios Rectores del Desarrollo Sostenible COMUNICACIÓN DE LA COMISIÓN AL CONSEJO Y AL PARLAMENTO EUROPEO, Bruselas, 25.5.2005

${ }^{57}$ Los títulos se recogen literalmente, adaptándolas a su aplicación a la edificación de Parte 3.- Participación en las iniciativas locales del Programa 21: Planes de acción local en favor de sostenibilidad en la CARTA DE LAS CIUDADES EUROPEAS HACIA SOSTENIBILIDAD (1994).
PRINCIPIO DE PRECAUCIÓN: ...y adoptar medidas preventivas ${ }^{56}$.

\section{LA IMPORTANCIA DEL PROCESO: APLICACIÓN A LA EDIFICACIÓN}

Si se establece que, para un desarrollo sostenible, sea preciso limitar la emisión de $\mathrm{CO}_{2}$, la extracción de madera por encima de su crecimiento vegetativo, evitar el incremento de los residuos o limitar la ocupación de todo el territorio con actuaciones antrópicas, por abordar algunas áreas de distinta naturaleza, su objetivo o los medios que son útiles a esta empresa no tienen su origen en el proceso edificatorio, sino que le vienen impuestos desde otras áreas sociales, científicas o tecnologías.

Esta dependencia exige conocer, proyectar, valorar y tomar decisiones con datos tradicionalmente ajenos a lo que se ha considerado su actividad. La forma en que se acerca esta información (principio de transparencia) a la toma de decisiones en edificación exige establecer fases, hitos y objetivos que permitan la aportación de datos, el análisis, la evaluación y la toma de decisiones consecuente con esta realidad.

Esto no significa, en mi opinión, que el proyecto sea necesariamente pluridisciplinar, sólo que es preciso considerar supuestos, como ya existen en otros ámbitos, que deben estar expuestos de tal forma que sean comprensibles y aplicables con seguridad en el ámbito de la edificación. La opción más eficaz puede ser en ocasiones la colaboración de varios profesionales, en otras, la revisión desde áreas diversas o la existencia de documentos de aplicación a edificación elaborados con la colaboración de otros profesionales,...

En cualquier caso el trabajo debe apoyarse en la investigación y exposición clara de los resultados de las soluciones propuestas para que la prescripción, la toma de decisiones integradas y la evaluación y seguimiento inherentes a la arquitectura y la ingeniería puedan realizarse.

Reflexionar sobre el proceso y el modo en que este se pone en práctica es la piedra angular para que la actividad edilicia colabore con un desarrollo sostenible, determinando no sólo el edificio sino también el resultado final: su adecuación, uso, mantenimiento,...

Para realizar una edificación de acuerdo con un desarrollo sostenible puede considerarse, como primera aproximación, un proceso similar al recomendado a los planes de acción local de la Agenda 21 (8), en Europa ${ }^{57}$.
Localización sistemática de los problemas y de sus causas mediante extensas consultas públicas

Dando prioridad a la satisfacción de las necesidades (principio de equidad y universalidad) frente a la creación de demanda (principio de autonomía), en relación a su ubicación (principio de localización), aportando información suficiente (principio de participación), estableciendo las causas de los procesos (principio de causalidad), observando las tendencias para relacionar las actividades con sus consecuencias (principio de precaución),...

Reconocimiento de los métodos de planificación y de los mecanismos financieros existentes, así como otros planes y programas

Compaginando la formación técnica, económica y social en los procesos para su puesta en práctica. Para ello será precisa una difusión adecuada de los resultados de actuaciones similares (principio de transparencia), la colaboración efectiva del conjunto de la comunidad para satisfacer objetivos colectivos (principio de corresponsabilidad), considerando las opciones que limitan los riesgos (principio de precaución), actuando especialmente sobre las causas de los problemas (principio de causalidad), confirmando su adaptación al entorno de la actuación y a sus capacidades (principio de localización),...

Creación de un modelo de comunidad sostenible mediante un proceso participativo que incluya a todos los sectores de la comunidad

Investigar procesos en los que pueda participar la totalidad de los agentes implicados, en todas las fases del proceso (principio de participación). Esto repercutirá en el desarrollo de modelos edilicios adaptados a las necesidades (principio de autonomía), hará posible reconocer la función de cada uno de los agentes, estableciendo los cauces para llevarla a cabo (principio de corresponsabilidad), ...

Clasificación de las tareas por orden de prioridad para tratar los problemas detectados

Las tareas, prescripciones, procesos, actividades en el ámbito de la edificación deben someterse a unas prioridades que cada sociedad habrá de establecer en función del itinerario elegido para conseguir su desarroIlo sostenible. Cumpliendo este itinerario la edificación habrá de cumplir prestaciones en base a exigencias que serán definidas por la sociedad en su conjunto (principio de parti- 
cipación), dando a cada conjunto de usuarios la posibilidad de definir sus prioridades (principio de autonomía), atendiendo a que las soluciones además de paliar los efectos, en su caso, atiendan a las causas de los problemas (principio de causalidad), actuando cada uno de los agentes en el ámbito de su autoridad para aportar las soluciones (principio de corresponsabilidad), sin olvidar el entorno concreto en que se producen las actuaciones (principio de localización), ni los riesgos que con las soluciones se contraen (principio de precaución),...

\section{Consideración y evaluación de opciones estratégicas alternativas}

Esto exige el desarrollo y puesta en uso de herramientas que permitan conocer la validez de las alternativas elegidas incorporando el modo en que se satisface esta necesidad (principio de transparencia). La actividad en edificación, especialmente en fase de proyecto, no puede por menos que someterse a evaluación para establecer alternativas que corrijan las consecuencias no deseadas de la actuación (principio de causalidad), alcanzar las prestaciones estimadas, y evitar riesgos (principio de precaución). Así podrá elegirse la opción más adecuada.

El análisis de actuaciones ya realizadas ha permitido determinar que las soluciones adecuadas a cada lugar y cada momento (principio de localización), en una determinada sociedad (principio de diversidad) puede exigir modificaciones en su desarrollo, como la necesidad de corrección de errores en las fases anteriores o para atender modificaciones sustanciales en la naturaleza social, económica o ambiental en el área geográfica donde se realizará la actuación. La consideración de alternativas y su análisis previo puede ser de enorme importancia para reducir las consecuencias de las modificaciones.

Establecimiento de un plan de acción local a largo plazo en favor de un desarrollo sostenible que incluya objetivos mensurables

Durante el proceso de proyecto, construcción, uso y al final de su vida útil será preciso establecer y comprobar el cumplimiento modal de los objetivos previstos, el los ámbitos social, económico y medioambiental (principio de participación),...

Programación de la aplicación del plan, incluida la preparación de un calendario y una declaración del reparto de responsabilidades entre los participantes

La planificación debe estar acompañada de una programación y reparto de responsabi- lidad proporcional a la autoridad, capacidades y recursos de cada agente (principio de corresponsabilidad),...

Establecimiento de sistemas y procedimientos para la supervisión y la notificación de la aplicación del plan

Merecen una especial atención los mecanismos para la supervisión y las actuaciones correctivas o de mantenimiento necesarias (principio de autonomía).

En cuanto a los mecanismos de supervisión la opinión mayoritaria es la de adoptar indicadores (principio de transparencia) como herramientas que permitan un análisis global y comparar los procesos en relación al entorno (estado del impacto humano sobre el entorno y el resto de los seres humanos, incluyendo su salud, su economía y sus relaciones sociales,...)

Las actuaciones correctivas y de mantenimiento están relacionadas con la vida útil del edificio. Por lo que este debe estar en una permanente revisión (mantenimiento) para que siga colaborando en la consecución de los objetivos marcados o adaptándose a sus sucesivas revisiones. Dentro de esta fase la difusión de resultados para alimentar el resto del proceso es ineludible.

El final de su vida útil debe estar prevista desde su origen y realizarse como una nueva actividad, en la que se consideren nuevamente las fases marcadas.

Como se ha indicado esquemáticamente, y sin carácter excluyente, en cada una de estas fases la consideración de los principios enunciados influye en la colaboración de las actuaciones en un desarrollo sostenible, de ahí el término de principio para el desarrollo sostenible que se ha adoptado para su definición en el texto.

\section{CONCLUSIÓN}

A pesar de la falta de acuerdo en este campo y las dificultades para establecer actividades colectivas para afrontar la satisfacción de las necesidades de los hombres, debe considerarse la importancia del modo en que se producen las actuaciones a la par que su resultado. Se propone la consideración y evaluación del camino elegido para conseguir los objetivos, en la convicción de que solo los actos, y no solo los logros, colaboran con el desarrollo sostenible. Esto puede invitar a considerar la incorporación de una estimación de su cumplimiento en cada una de las fases del proceso, desde la determinación de los objetivos a su evaluación, pasando por la definición de los indicadores, la elección 
58 Se insiste en la denominación de principios frente a la de valores, por la connotación de este último término como cualidad, grado de utilidad para satisfacer las necesidades, proporcionar bienestar o deleite, $y$, por ello de ser susceptible de ser medidos, mientras que a los principios se consideran causa, razón fundamental, norma o idea que rige una conducta, un hecho, un pensamiento o resulta responsable de las propiedades de una sustancia, afectando a orden social, ambiental y económico.

${ }^{59}$ Se han considerado, entre otros, los trabajos para "Indicators for Sustainable Development: Theory, Method, Applications. A Report to the Balaton Group" Bossel, $\mathrm{H}$. Winnipeg, 1999 y RENEWED EU SUSTAINABLE DEVELOPMENT STRATEGY. COUNCIL OF THE EUROPEAN UNION 10117/06 Bruselas, 2006.

${ }^{60}$ Es pertinente recordar aquí, más para indicar el camino que aún queda por recorrer y establecer las limitaciones de este trabajo con relación a la referencia que se cita, la afirmación "escogido el tema de estudio...entre los procedimientos de estudio se escogerán los mas recientes, y sobre todo los más difíciles, ...los resultados obtenidos tendrán gran importancia y nos indemnizarán con creces de nuestros afanes" (S. Ramón y Cajal, 1897). Aplicada a las ciencias biológicas se encuentra un paralelo para su aplicación a las ciencias sociales y económicas que competen, también, al desarrollo sostenible. de la tecnología apropiada, prescripción de materiales y equipos, procedimientos de actuación,... Esta visión compleja permitirá indicar claramente las tendencias, los progresos y las regresiones en la consecución del objetivo marcado.

Se propone considerar, en cada indicador empleado en su caracterización, cada uno de aquellos principios ${ }^{58}$ que son inherentes al desarrollo sostenible, ya que ni en su adición ni en su consideración por separado, garantiza obtener buenos resultados, aportando solo una información sesgada y de baja calidad informativa ${ }^{59}$ (9).

En la edificación desde la gestación en el proyecto y la definición de objetivos, durante la definición constructiva y la evaluación del cumplimiento de los objetivos, durante la puesta en obra y comprobación del cumplimiento de los objetivos, durante su uso y mantenimiento, monitorización y difusión de los resultados,....se deben considerar aquellos principios que le permiten colaborar en un desarrollo sostenible. Solo entonces será correcto el empleo de denominaciones como construcción sostenible, arquitectura sostenible,...como una contracción de "construcción para un desarrollo sostenible" o "arquitectura para un desarroIlo sostenible".

Es importante ser consciente de la dificultad de aplicación a que se somete al proceso y la necesidad de reconsiderar el trabajo realizado, aunque no se considera un eximente para iniciar este camino; por otra parte, común a todas las actividades que tienen entre sus objetivos una aplicación práctica y no sólo el enunciado de las ideas ${ }^{60}(10)$.

Esta concepción global aconseja la elección para la edificación de tecnologías, usos, procedimientos, prácticas,... de eficiencia energética, ahorro de agua, recuperación y uso de aguas utilizadas o pluviales, reciclaje de residuos, empleo de materiales sanos, análisis del ciclo de vida, limitación de la cantidad de material, construcción eficiente, bioclimatismo,...explorando sus interferencias y su colaboración efectiva. Siendo conscientes de que la adición o el uso indiscriminado de estos recursos, instrumentos o herramientas no garantizan la colaboración con el fin previsto.

Se propone, en resumen, aplicar a la edificación este carácter modal. Esto exige definir las actuaciones en cada una de sus fases, atendiendo a la singularidad del lugar y a su adaptación a los fines que se pretenden. Así como respetar las fases, hitos y comprobaciones inherentes a la actuación, la información y difusión pública de sus resultados,... atendiendo a todo el proceso y no solamente a sus logros.

\section{AGRADECIMIENTO}

A los Alumnos de la Disciplina de Iniciación a la Construcción Sostenible de la Escuela de Arquitectura Técnica de la Universidad Politécnica de Madrid que, con sus aportaciones, han colaborado a definir durante estos últimos cinco años las bases para una actuación técnica que colabore a un desarrollo sostenible.

\section{BIBLIOGRAFÍA}

(1) Our Common Future, Chapter 2: Towards Sustainable Development. From A/42/427. Our Common Future: Report of the World Commission on Environment and Development.1. Chairman Gro Harlem Brundtland Oslo, 20 March 1987.

(2) Fromm, E : The Fear of Freedom, H. Brown. Routledge, London, 1941.

(3) Goodland, R and Daly, H, Environmental Sustainability: Universal and Non-Negotiable, Ecological Applications, Vol. 6, No. 4 (Nov., 1996), pp. 1002-1017.

(4) Commoner, B. The Closing Circle. Nature, Man \& Technology. New York: Bantam Books. (1971)

(5) Proyecto de Declaración sobre los Principios Rectores del Desarrollo Sostenible. Comunicación de la Comisión al Consejo y al Parlamento Europeo, Bruselas, 25.5.2005.

(6) Las definiciones están tomadas de la versión 22 del diccionario de la R.A.E.

(7) Declaración Universal de los Derechos Humanos, 1948.

- Declaración de Estocolmo sobre el medio humano. Conferencia de Naciones Unidas sobre el Medio Humano, Estocolmo del 5 al 16 de junio de 1972.

- Declaración de Río sobre el Medio Ambiente y el Desarrollo. Conferencia de las Naciones Unidas sobre el Medio Ambiente y el Desarrollo, Río de Janeiro del 3 al 14 de junio de 1992.

Tratado de la Unión Europea Diario Oficial n C 191 de 29 de julio de 1992 (1992) Titulo XVI MEDIO AMBIENTE.

- PACTO MUNDIAL. RESOLUCIÓN SOBRE LA INCLUSIÓN DE UN DÉCIMO PRINCIPIO CONTRA LA CORRUPCIÓN 30 DE MAYO DE 2004, propuesta del Secretario General de la ONU Kofi Annan. 
- Proyecto de Declaración sobre los Principios Rectores del Desarrollo Sostenible COMUNICACIÓN DE LA COMISIÓN AL CONSEJO Y AL PARLAMENTO EUROPEO, Bruselas, 25.5.2005.

- La posición Europea actualizada se recoge en 9.2.2005 "COMUNICACIÓN DE LA COMISIÓN AL CONSEJO Y AL PARLAMENTO EUROPEO Revisión en 2005 de la Estrategia de la Unión Europea para un desarrollo sostenible: Primer balance y orientaciones futuras 5.5.

TRATADO DE NIZA. CONSEJO EUROPEO DE NIZA 7-10 de diciembre de 2000 CONCLUSIONES DE LA PRESIDENCIA.

Tratado de la Unión Europea (1992).

(8) CARTA DE LAS CIUDADES EUROPEAS HACIA SOSTENIBILIDAD (1994). Parte 3.- Participación en las iniciativas locales del Programa 21: Planes de acción local en favor de sostenibilidad.

(9) "Indicators for Sustainable Development: Theory, Method, Applications. A Report to the Balaton Group" Bossel,H. Winnipeg, 1999 y RENEWED EU SUSTAINABLE DEVELOPMENT STRATEGY. COUNCIL OF THE EUROPEAN UNION 10117/06 Bruselas, 2006.

(10) S. Ramón y Cajal. Reglas y consejos sobre investigación científica. Librería Beltrán, Madrid, 1897. 